\title{
LINKAGE STUDY OF DOMINANTLY INHERITED OLIVO-PONTO-CEREBELLAR ATROPHY (OPCA) AND HOLMES' ATAXIA
}

\author{
Hidenao SASAKI, ${ }^{2}$ Akemi WAKISAKa, ${ }^{2}$ Toshiaki KarOH, ${ }^{3}$ \\ Michihiro C. Yoshida, ${ }^{4}$ Takeshi Hamada, ${ }^{5}$ Kohji Shima, ${ }^{6}$ \\ Tohru MatsuUra, ${ }^{1}$ and Kunio Tashiro ${ }^{1}$ \\ ${ }^{1}$ Department of Neurology and "Department of Pathology, School of Medicine, \\ Hokkaido University, Sapporo 060: ${ }^{3}$ Hokkaido Red Cross Blood Center, \\ Sapporo 060; 'Chromosomal Research Unit, Faculty of Science, \\ Hokkaido University, Sapporo 060; ${ }^{5}$ Hokuyukai Neurological Hospital, \\ Sapporo 060; ${ }^{6}$ Section of Neurology, Sapporo Minami National \\ Sanatory Hospital, Sapporo 060, Japan
}

Summary Both autosomal dominant olivo-ponto-cerebellar atrophy (OPCA) and Holmes' ataxia are the progressive neurodegenerative disorders of adulthood with unknown biochemical defects. In order to determine the genetic locus and possible genetic heterogeneity, linkage study was performed in 19 OPCA families comprising 180 individuals with 60 affected patients, and two Holmes' ataxia families comprising 39 individuals with 10 affected patients. By using computer program LIPED, linkage of each disorder was analyzed to 12 blood groups, 5 red cell enzymes, HLA-A, -B, -C, and F13A. No evidence suggesting linkage to these two disorders was obtained in the markers examined, including three $6 \mathrm{p}$ markers such as HLA, GLO1, and F13A. Furthermore, in 14 out of 15 HLAinformative OPCA families, negative lod scores for OPCA with HLA were obtained at most recombination fractions. Our results provide further evidence suggesting the genetic heterogeneity of dominant OPCA.

Key Words spinocerebellar ataxia, HLA, linkage study, Holmes' ataxia, olivo-ponto-cerebellar atrophy

\section{INTRODUCTION}

Idiopathic hereditary spinocerebellar degeneration or ataxia (SCA) is a cluster

Received September 5, 1988; revised version received October 8, 1988; Accepted October 28, 1988 Address correspondence and reprint request to: Hidenao Sasaki, M.D., Department of Neurology, School of Medicine, Hokkaido University, N-14, W-5, Kita-ku, Sapporo 060, Japan. 
of neurodegenerative disorders. Of these disorders, hereditary olivo-ponto-cerebellar atrophy (OPCA Menzel type) and Holmes' hereditary cerebello-olivary degeneration (Holmes' ataxia) are the main disorders showing autosomal dominant inheritance with a relatively late age of onset (usually fourth to fifth decades). However, the clinical features and course, and the distribution of pathological alteration are quite different from each other, and these two disorders are considered to be genetically different entities. Main clinical manifestations of dominant OPCA are cerebellar ataxia associated with limb spasticity, amyotrophy, and sings and symptoms of cranial nerves and basal ganglia involvements. Pathologically, neuronal degeneration and gliosis are observed in cerebellar cortex, deep cerebellar nuclei, brain stem, spinal cord, and basal ganglia. In contrast, Holmes' ataxia shows only cerebellar ataxia, and pathological alterations are confined to cerebellar cortex and olivary nucleus (Harding, 1984). Therefore, clinical differentiation between OPCA and Holmes's ataxia is possible with considerable certainty during life.

In both OPCA and Holmes' ataxia, little is known about biochemical defects. The causing genes for OPCA and Holmes' ataxia are not known but the first possible linkage of OPCA was reported with HLA markers on the short arm of chromosome 6 (Yakura et al., 1974). After their report, Jackson et al. (1977) reported the evidence that OPCA locus is located at $12 \mathrm{cM}$ apart from HLA locus. In subsequent studies, the linkage has been investigated between OPCA and genetic markers on 6p (Jackson et al., 1978; Möller et al., 1978; Wastiaux et al., 1978; Pedersen et al., 1980; Nino et al., 1980; Whittington et al., 1980; Morton et al., 1980; Koeppen et al., 1981; Haines et al., 1984; Haines and Trofatter 1986; Kumar et al., 1986; Rich et al., 1987; Wilkie et al., 1987; Kanazawa et al., 1987; Zoghbi et al., 1987, 1988). These data show that the locus for OPCA is on 6p24-p21.3 (The first OPCA locus mapped to $6 \mathrm{p}$ has been now defined as SCA1. See HGM9; Olaisen et al., 1987). However, linkage analysis on additional families is necessary to determine if there is more than one OPCA locus, and whether the Holmes' ataxia is linked to HLA. Since the existence of genetic heterogeneity would be important for future diagnosis, isolation and characterization of the OPCA and Holmes' ataxia genes, we have begun genotyping a large collection of OPCA and Holmes' ataxia families. In this paper, we report the preliminary results of genotyping 19 OPCA and 2 Holmes' ataxia families with HLA and other genetic loci. Our data provide further evidence suggesting the existence of genetic heterogeneity of dominant OPCA. In addition, this is the first report for a linkage study of Holmes' ataxia.

\section{MATERIALS AND METHODS}

Family resources. The 21 OPCA and Holmes' ataxia affected families used in this study were contributed by the Department of Neurology of Hokkaido University School of Medicine, Hokuyukai Neurological Hospital, Sapporo Minami National Sanatory Hospital, and Kushiro Rosai Hospital. Data of the date of birth, age 
of onset of the disease, and the number of children were collected in those families as much as possible. All of the patients were examined by one of the authors (H.S.). The determination of clinical diagnosis is based on the positive family history, typical clinical manifestations of the affected members, and pathognomonic alterations of brainstem and cerebellar atrophies observed on X-ray computed tomography or magnetic resonance imaging. The clinical diagnosis has been confirmed pathologically in 2 families, P.2 and P.16. Clinico-pathological features of autopsied cases in these 2 families have been reported by Fujii et al. (1987) and Kudoh et al. (1986), respectively. In the other 17 OPCA families and 2 families of Holmes' ataxia, the clinical diagnosis has not been confirmed pathologically yet.

Taking the possible heterogeneity of OPCA into account, differential diagnosis of OPCA from other related disorders were carefully performed. In our OPCA patients, no cases manifesting dystonia were observed, so far examined. Therefore, Joseph disease, a clinical subtype of dominant OPCA (Rosenberg, 1986), was not included in the present OPCA families. Dentato-rubro-pallido-Luysian atrophy (DRPLA), particularly ataxochoreoathetoid form, was the another disorder which must be differentiated from dominant OPCA. In this regard, it has been known that choreic or athetotic involuntary movement is occasionally observed in hereditary OPCA (Harding, 1984). In the present OPCA families, 2 cases in family P. 2 showed choreic movement. However, cerebellar ataxia and pyramidal tract involvement predominate the clinical pictures of the other patients of this family, which is consistent with the diagnosis of hereditary OPCA. Overall, the clinical features of the present patients were within the clinical spectrum of dominant OPCA.

The pedigrees were compiled on the basis of informations provided by extensive search for family members. It was left "uncertain" when the mode of inheritance could not be established due to the early death of parent. The affected families have now been continuously collected. In this report, the families available of linkage informations were analyzed. These 19 OPCA families comprised 180 individuals, with 60 affected subjects. The two Holmes' ataxia families comprised 39 individuals with 10 affected patients (Table 1 and Fig. 1). The pedigree data of these 21 SCA families (P.1 to P.21) were now available, spanning 6 generations in the largest pedigree (P.1), and 3 generations in the small ones.

Determination of genetic markers. Blood samples were collected from each individual for determination of genetic markers and lymphoblastoid cell transformation. The genetic markers examined in this study and their current chromosomal assignments were presented in Table 2 .

Blood-group typing. After blood sampling, the 12 blood groups were serotyped using standard methods. They include $\mathrm{ABO}, \mathrm{Rh}(\mathrm{CcDdEe})$, Lewis, MNSs, Diego, Kell, Duffy, Kidd, Lutheran, $\mathrm{Xg}$, Jr, and $\mathrm{P}$.

$H L A$ typing. The standard NIH microlymphocytotoxicity test was used for HLA-A, -B, -C typing.

Enzymes. Hemolysates, stored in a deep freezer, were used as a sample for 
Table 1. Families affected with hereditary spinocerebellar ataxia.

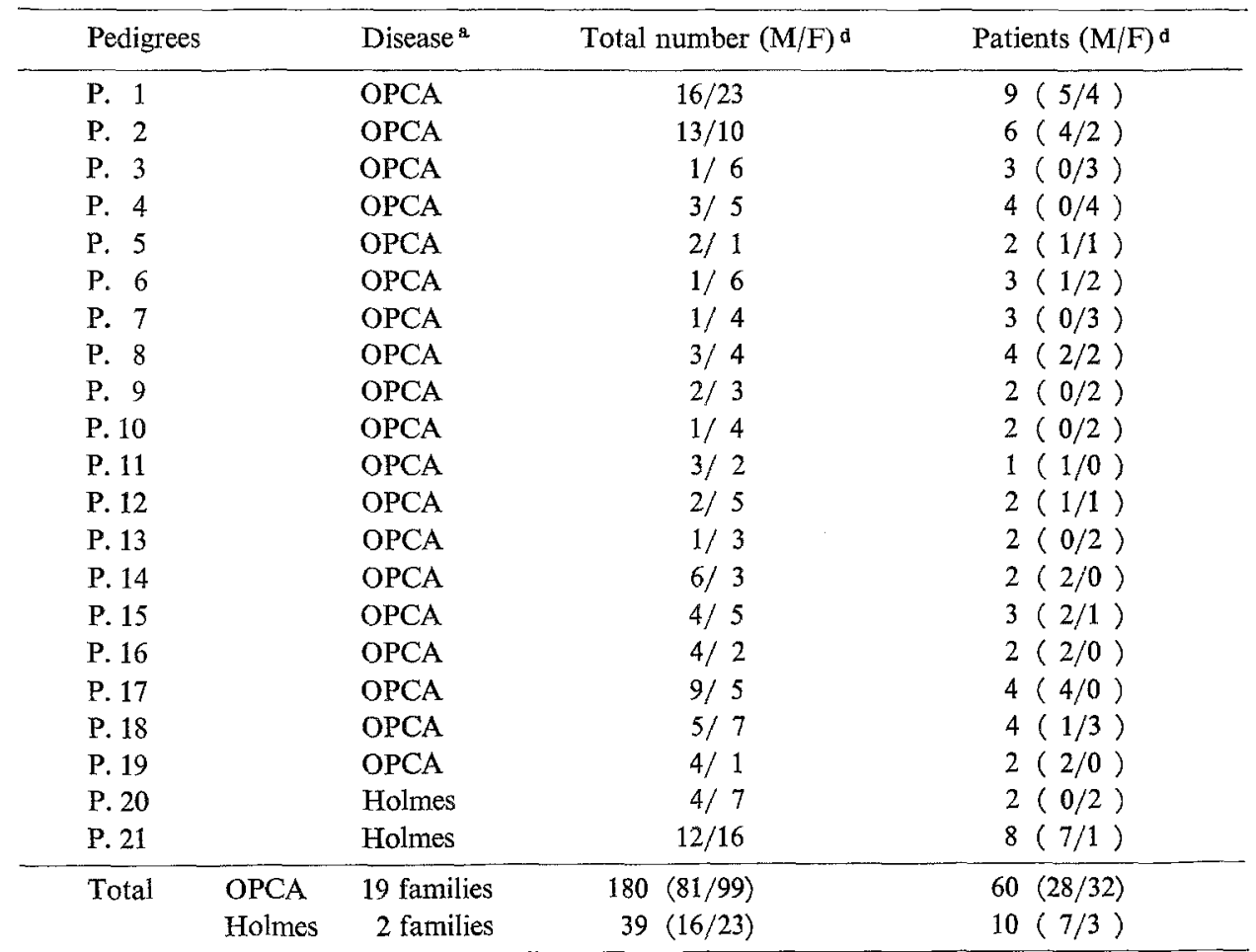

' OPCA, olivo-ponto-cerebellar atrophy (Menzel type); Holmes' ataxia, Holmes' hereditary cerebello-olivary atrophy. b $\mathrm{M}$, male; F, female.

Table 2. Genetic markers used for linkage study.

\begin{tabular}{lc}
\hline Marker & Chromosomal assignment $^{\mathrm{a}}$ \\
\hline ABO & $9 \mathrm{q} 34.1-\mathrm{q} 34.2$ \\
Rh & $1 \mathrm{p} 36.2-\mathrm{p} 34$ \\
MNS & $4 \mathrm{q} 28-\mathrm{q} 31$ \\
Diego & unknown \\
Kidd(Jk) & $18 \mathrm{q} 11-\mathrm{q} 12$ \\
Duffy(Fy) & $1 \mathrm{p} 21-\mathrm{q} 23$ \\
P & 6 \\
AcP1 & $2 \mathrm{p} 25$ or $\mathrm{p} 23$ \\
EsD & $13 \mathrm{q} 14$ \\
6PGD & $1 \mathrm{p} 36.2-\mathrm{p} 36.13$ \\
GLO1 & $6 \mathrm{p} 21.3-\mathrm{p} 21.1$ \\
PGM1 & $1 \mathrm{p} 22.1$ \\
HLA & $6 \mathrm{p} 21.3$ \\
F13A & $6 \mathrm{p} 24-\mathrm{p} 21.3$ \\
(SCA1) & $(6 \mathrm{p} 24-\mathrm{p} 21.3)$ \\
\hline
\end{tabular}

\footnotetext{
a From HGM9.
} 


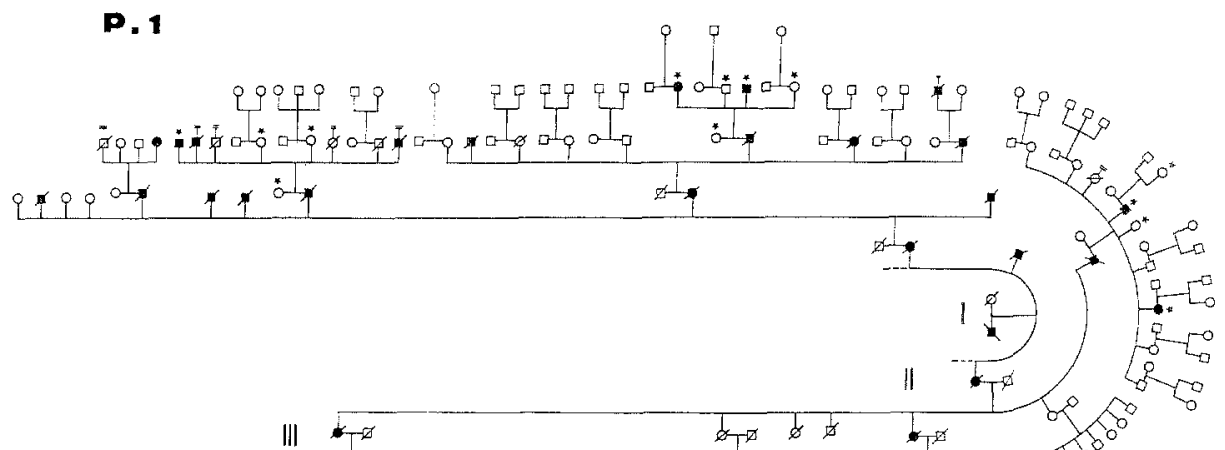

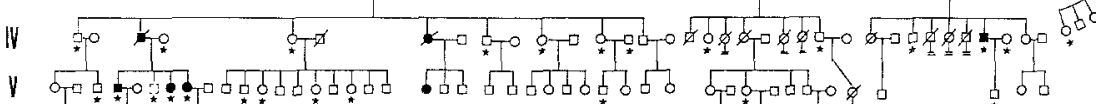

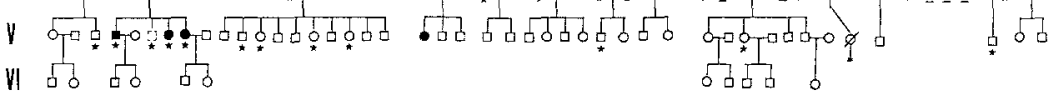

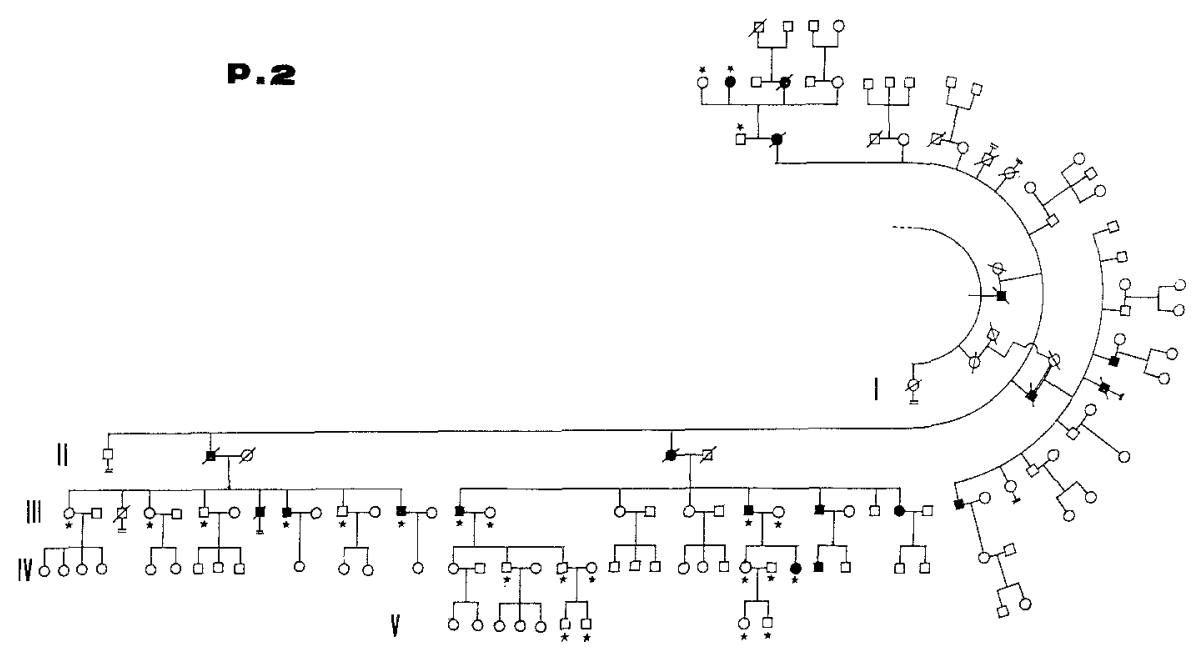

\section{P. $\mathbf{B}$}

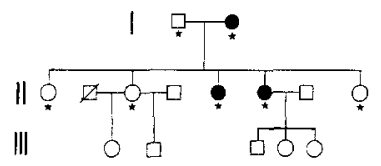

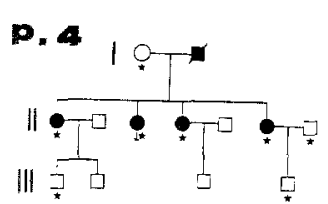

P. E

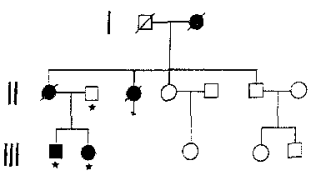

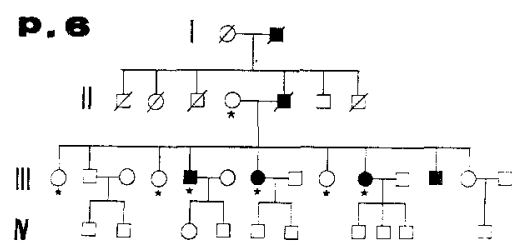

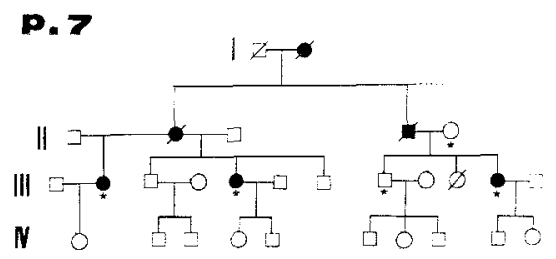

Vol. 33, No. 4, 1988 
P. 8

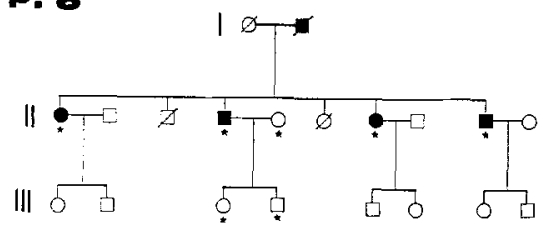

P. 9

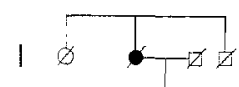

II
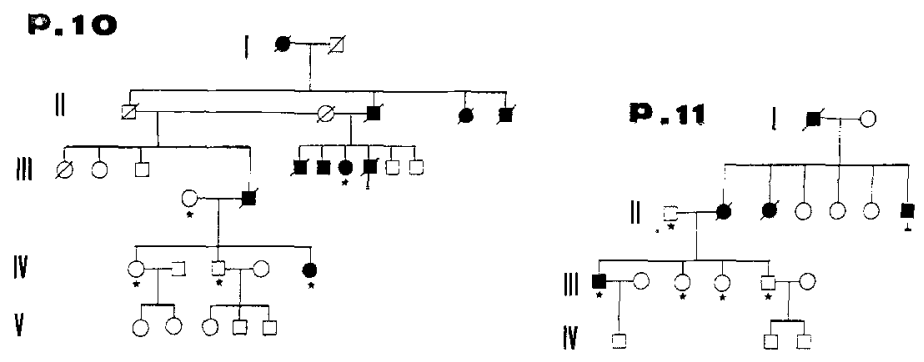

P.12

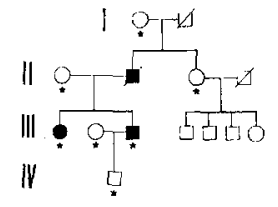

\section{P. 13}

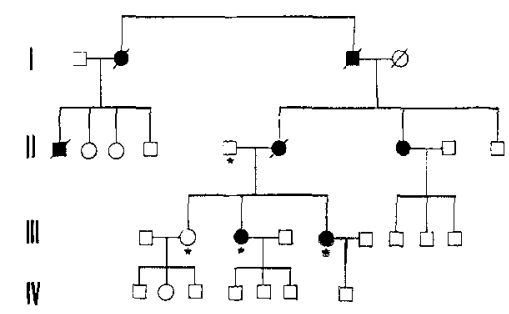

P. 16

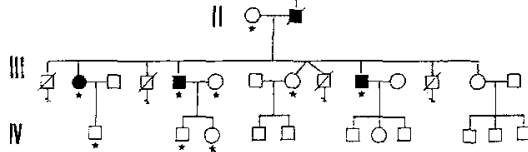

p.17

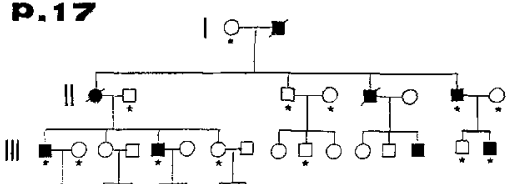

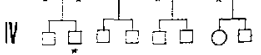
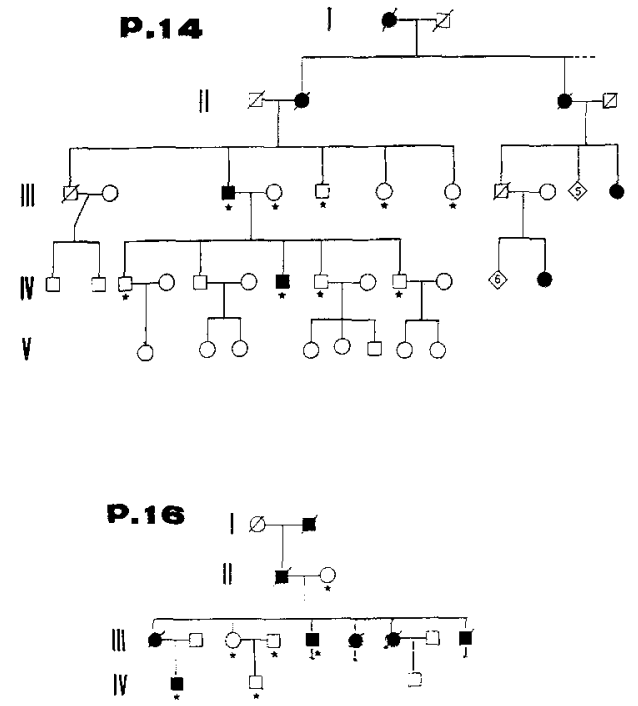

P.18

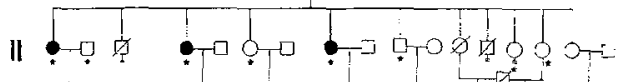

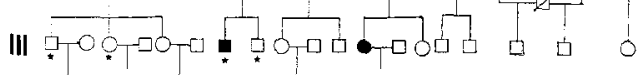

iv 1500 口过 

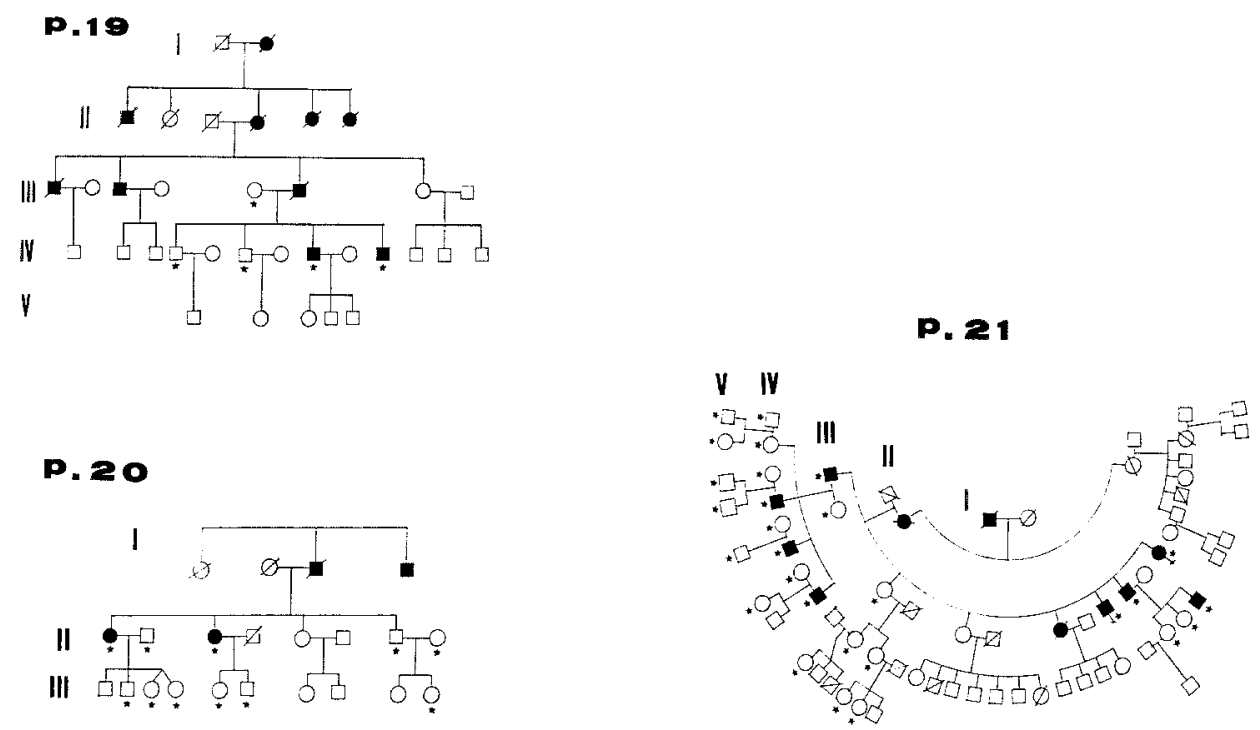

\section{P. 20}

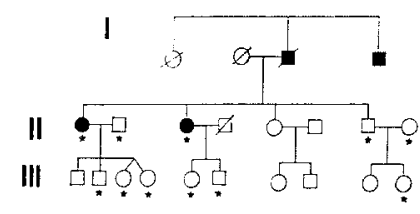

Fig. 1. The family pedigrees of dominant spinocerebellar ataxia. The deduced pedigrees of 21 different families with dominant ataxias are presented. P. 1 to P. 19 are the families with dominant OPCA. P. 20 and P. 21 with Holmes' ataxia. In the pedigrees, generations are expressed as Roman numerals. Deceased individuals are shown by slash. Males are indicated by squares, females by circles, and affected patients are shown by filled in symbols. Individuals examined in this investigation are indicated with filled stars.

enzyme electrophoresis. Isoenzymes of glyoxalase-1 (GLO1), esterase D (EsD), 6-phosphogluconate dehydrogenase (6PGD), red cell acid phosphatase (AcP1), were analyzed by starch gel electrophoresis, as described by Harris and Hopkinson (1976). Phosphoglucomutase-1 (PGM1) was examined by the isoelectric instrument focusing method of the manual of LKB (Sweden).

Coagulating factor XIIIA (FI3A). According to the monodansyl cadaverinecasein functional detection method of Board (1979), polymorphism of F13A was determined after high-voltage agarose gel electrophoresis of plasma samples.

Linkage analysis. Linkage analysis was performed using the computer program LIPED with the correction of age-dependent penetrance (Ott, 1974); the lod score over +3 is regarded as a proof of close linkage, while the value below -2 is strong evidence of non-linkage at given recombination fractions. In case of the HLA antigen, haplotypes were scored as a single unit, according to the instruction of Ott (1978). Because of the dominant trait of these disorders, it was assumed that all the patients were heterozygotes. The age of onset was defined as the age when the patient first noticed clinical symptoms. Mean \pm SD of the age at onset were 
inputted at computation of the lod scores of hereditary OPCA and Holmes' ataxia, respectively. The penetrance was assumed to be $100 \%$.

\section{RESULTS}

\section{Pedigree data}

Nineteen families with hereditary OPCA and 2 families with Holmes' ataxia were selected for genetic linkage analyses (Table 1 and Fig. 1). The 19 OPCA families (P.1 to P.19) comprised 180 individuals, including 60 affected patients who are still alive. The 2 families with Holmes' ataxia (P.20 and P.21) comprised 39 individuals, with 10 affected subjects. Interrelationship within these family pedigrees is anknown at present.

Major clinical features of the affected members in each OPCA family were fairly uniform with cerebellar ataxia, nystagmus, hyperactive deep tendon reflexes, and positive Babinski and/or Chaddock signs. Facial and/or lingual muscle twitching, neck flexor weakness, optic disc pallor, mydriasis, upward gaze palsy, dysphagia, limb spasticity, limb muscle atrophy, or impaired vibratory perception were also observed in parts of advanced patients. In four families, P.2, P.11, P.12, and P.16, characteristic disturbance of pursuit eye movement, known as "slow eye movement" or slow saccade, was observed in parts of affected patients. Two patients in P.2 showed choreic movement, and one of them showed marked neurogenic limb muscle atrophy. Rhythmic myoclonus was observed in a case of family P.2 and P.12, respectively.

As shown in Table 1 and Fig. 1, affected subjects were distributed throughout more than two generations in both OPCA and Holmes' ataxia. There were 28 males and 32 females affected by OPCA (male : female ratio $=0.88$ ), and 7 male and 3 female by Holmes' ataxia (ratio $=2.3$ ). In every generation, each family had affected individuals except for those younger than the age of onset or dead with non-neurological diseases or by accident. Of informed patients, mean age of onset was $32.7 \pm 10.3(\mathrm{n}=57$, ranged from 17 to 56 years old $)$ in OPCA, and $36.0 \pm$ 5.4 ( $n=9$, ranged from 26 to 46 years old) in Holmes' ataxia. This fact implies that most of carriers manifest symptoms after the critical age at onset. This mode of inheritance, no sexual preference, and the age of onset clearly indicate that these two ataxic disorders are inherited in an autosomal dominant trait with age-dependent penetrance.

\section{Linkage analysis of OPCA}

Genotyping 19 OPCA-affected families, comprising 180 individuals, resulted in 15 families informative for HLA markers. The results of the OPCA-HLA linkage analysis are presented in Table 3. The maximum lod score was $0.868(\theta=0.000)$ in P.16. In the other families, even in two large families (P.1 and P.2), negative lod scores were found at most recombination fractions. These results demonstrated no 
Table 3. Lod score for OPCA and HLA loci.

\begin{tabular}{|c|c|c|c|c|c|c|}
\hline \multirow{2}{*}{ Pedigree } & \multicolumn{6}{|c|}{ Recombination fraction $(\theta \mathrm{mf})^{a}$} \\
\hline & 0.000 & 0.050 & 0.100 & 0.200 & 0.300 & 0.400 \\
\hline P. $1(68)^{b}$ & -14.400 & -2.117 & -1.114 & -0.224 & 0.098 & 0.141 \\
\hline P. $2(30)$ & -19.348 & -4.379 & -2.994 & -1.584 & -0.806 & -0.315 \\
\hline P. $3(7)$ & -4.562 & -0.844 & -0.538 & -0.244 & -0.097 & -0.023 \\
\hline P. $4(10)$ & -3.026 & -0.411 & -0.182 & -0.025 & 0.013 & 0.011 \\
\hline P. $5(4)$ & -3.683 & -0.721 & -0.444 & -0.194 & -0.076 & -0.018 \\
\hline P. $7(4)$ & -0.648 & -0.429 & -0.298 & -0.142 & -0.058 & -0.014 \\
\hline P. $8(9)$ & -3.100 & -1.438 & -0.889 & -0.390 & -0.153 & -0.037 \\
\hline P. 10 (9) & -0.108 & -0.085 & -0.066 & -0.036 & -0.016 & -0.004 \\
\hline P. $11(6)$ & -1.317 & -0.575 & -0.350 & -0.147 & -0.055 & -0.127 \\
\hline P. $12(9)$ & -8.032 & -1.746 & -1.186 & -0.664 & -0.380 & -0.177 \\
\hline P. $14(11)$ & -2.799 & -0.891 & -0.419 & -0.021 & 0.112 & 0.108 \\
\hline P. 15 (13) & -0.273 & -0.148 & -0.078 & -0.018 & -0.006 & -0.006 \\
\hline P. 16 (9) & 0.868 & 0.782 & 0.691 & 0.499 & 0.302 & 0.124 \\
\hline P. $17(16)$ & -11.918 & -1.125 & -0.589 & -0.137 & 0.035 & 0.069 \\
\hline P. $18(15)$ & -9.332 & -3.014 & -2.064 & -1.071 & -0.519 & -0.185 \\
\hline Total & -81.677 & -17.141 & -10.521 & -4.399 & -1.605 & -0.337 \\
\hline
\end{tabular}

a $\theta \mathrm{mf}$, sex-combined lod score. b Number within parenthesis indicates the total members of the family; it includes both the members alive and the deceased ones whose genotypes are deduced from family pedigree.

Table 4. Sex-specified lod scores for F13A:HLA, F13A:OPCA, and HLA:OPCA linkages.

\begin{tabular}{|c|c|c|c|c|c|}
\hline \multirow{2}{*}{ Locus pair } & \multicolumn{5}{|c|}{ Recombination fraction $(\theta)$} \\
\hline & 0.050 & 0.100 & 0.200 & 0.300 & 0.400 \\
\hline \multicolumn{6}{|l|}{ Paternal lod score } \\
\hline F13A:HLA (14) ${ }^{a}$ & -1.435 & -0.072 & 0.697 & 0.615 & 0.258 \\
\hline F13A:OPCA (11) & -1.667 & -1.033 & -0.356 & -0.034 & 0.068 \\
\hline HLA:OPCA (12) & -11.840 & -7.530 & -3.346 & -1.299 & -0.294 \\
\hline \multicolumn{6}{|l|}{ Maternal lod score } \\
\hline F13A:HLA (13) & -4.400 & -2.592 & -1.014 & -0.377 & -0.111 \\
\hline F13A:OPCA (8) & -0.543 & -0.173 & 0.150 & 0.163 & 0.060 \\
\hline HLA:OPCA (10) & -5.301 & -2.991 & -1.053 & -0.307 & -0.043 \\
\hline
\end{tabular}

a Number of informative pedigrees.

evidence of close linkage between the gene causing OPCA and HLA gene. When the lod scores of all pedigrees were pooled, OPCA-HLA linkage was excluded at the recombination fraction from 0.000 to 2.500 . 
Since the SCAI locus is assigned telomeric to HLA locus and in order to validate the procedure, paternal lod score $(\theta \mathrm{m})$ and maternal lod score $(\theta \mathrm{f})$ of F13A:OPCA, HLA:OPCA, and F13A:HLA linkage were calculated, respectively. As shown in Table 4, paternal F13A:HLA lod scores were positive, but not so high to support linkage; maternal F13A:HLA lod scores were negative at all recombination fractions. Slightly positive values were obtained in F13A:OPCA lod score, but the peak values of both sexes were not significant. Both maternal and paternal lod scores for HLA: OPCA linkage were negative at all recombination fractions. In paternal lod scores, the lod scores for HLA:OPCA linkage were far negative than that for F13A:HLA linkage throughout all recombination fractions.

In other genetic loci shown in Table 5, the lod scores for ABO, Diego, Jk, Fy, AcP1, 6PGD, and GLO1 were negative throughout all recombination fractions, whereas the lod scores for linkage between OPCA and Rh, MNS, P, EsD, PGM1, and F13A were slightly positive at higher recombination fractions, but the values were too small to suggest linkage between OPCA and these loci. These genotyping data showed no conclusive evident for linkage of OPCA to these 14 genetic loci, even to GLOI and F13A on 6p.

\section{Linkage analysis of Holmes' ataxia}

As shown in Table 6, the two families of Holmes' ataxia (P.20 and P.21), showed negative lod scores for all recombination fractions in ABO, MNS, Diego, GLO1, 6PGD, EsD, and HLA. Slightly positive lod scores were found versus P, PGM1,

Table 5. Lod scores between OPCA and 13 markers.

\begin{tabular}{lrrrrrr}
\hline & \multicolumn{5}{c}{ Recombination fraction $(\theta \mathrm{mf})^{\mathrm{a}}$} \\
\cline { 2 - 7 } Marker & 0.000 & 0.050 & 0.100 & 0.200 & 0.300 & \multicolumn{1}{c}{0.400} \\
\hline ABO & -18.504 & -3.485 & -2.141 & -0.869 & -0.298 & -0.065 \\
Rh & -14.651 & -1.589 & -0.602 & 0.016 & 0.097 & 0.051 \\
MNS & -28.351 & -3.644 & -2.141 & -0.765 & -0.159 & 0.054 \\
Diego & -15.158 & -3.731 & -2.113 & -0.639 & -0.130 & -0.024 \\
Jk & -18.215 & -2.866 & -1.715 & -0.697 & -0.232 & -0.028 \\
Fy & -6.631 & -0.719 & -0.413 & -0.123 & -0.014 & -0.005 \\
P & -14.357 & -1.000 & -0.283 & 0.203 & 0.265 & 0.150 \\
AcP1 & -44.560 & -7.591 & -4.851 & -2.195 & -0.894 & -0.251 \\
EsD & -11.840 & -1.130 & -0.376 & 0.085 & 0.102 & 0.010 \\
6PGD & -4.470 & -0.717 & -0.479 & -0.241 & -0.119 & -0.055 \\
GLO1 & -26.517 & -3.466 & -1.717 & -0.450 & -0.109 & -0.060 \\
PGM1 & -29.135 & -3.671 & -1.641 & -0.125 & 0.200 & 0.110 \\
F13A & -9.988 & -2.288 & -1.204 & -0.182 & 0.142 & 0.132 \\
\hline
\end{tabular}

a $\theta \mathrm{mf}$, sex-combined lod score. 
Table 6. Lod scores between Holmes' ataxia and 12 markers.

\begin{tabular}{lrrrrrr}
\hline & \multicolumn{5}{c}{ Recombination fraction $(\theta \mathrm{mf})^{\mathrm{a}}$} & \\
\cline { 2 - 6 } Marker & 0.000 & 0.050 & 0.100 & 0.200 & 0.300 & 0.400 \\
HLA & -15.962 & -4.763 & -3.083 & -1.510 & -0.706 & -0.245 \\
ABO & -7.858 & -1.761 & -1.004 & -0.383 & -0.133 & -0.245 \\
MNS & -0.691 & -0.496 & -0.355 & -0.172 & -0.069 & -0.016 \\
Jk & 0.622 & 0.552 & 0.478 & 0.325 & 0.173 & 0.050 \\
P & -0.039 & -0.025 & -0.014 & -0.003 & 0.000 & 0.000 \\
Diego & -0.549 & -0.283 & -0.161 & -0.053 & -0.015 & -0.003 \\
GLO1 & -0.238 & -0.185 & -0.141 & -0.075 & -0.032 & -0.008 \\
PGM1 & -4.953 & -0.327 & -0.111 & 0.016 & 0.027 & 0.010 \\
6PGD & -5.365 & -1.075 & -0.602 & -0.180 & -0.039 & -0.004 \\
EsD & -7.919 & -2.228 & -1.387 & -0.613 & -0.241 & -0.057 \\
AcP1 & 0.121 & 0.102 & 0.084 & 0.050 & 0.024 & 0.006 \\
F13A & 0.001 & 0.001 & 0.003 & 0.002 & 0.001 & 0.000 \\
\hline
\end{tabular}

a $\theta \mathrm{mf}$, sex-combined lod score.

AcP1, Jk, and F13A. These data showed no evidence of linkage between Holmes" ataxia and HLA and other loci here examined.

\section{Clinical difference between HLA-linked and HLA-nonlinked OPCA}

If any of HLA-linked OPCA were included in the present 15 HLA-informative OPCA families, only P.16 was likely to be linked to HLA. However, the pedigree size and the number of individuals of P.16 was so small that it was impossible to discriminate true HLA-OPCA linkage from HLA haplotype segregation by chance. In this family, 2 patients examined by one of the authors (H.S.) and other cases reported by Kudoh et al. (1986) clinically manifested slow eye movement, limb muscle atrophy, positive Babinski and/or Chaddock sign with minor leg spasticity, and terminal mental decline. However, these clinical features were also observed in the part of patients of HLA-nonlinked families, such as P.2, P.11, and P.12. The predominant clinical picture of other HLA-nonlinked families was rather uniform, showing dysarthria, nystagmus, dysphagia, ataxia, and leg spasticity with positive Babinski and/or Chaddock sign. From these available information, we have not found any clinical difference between the two type of OPCA families.

\section{DISCUSSION}

Determination of each gene causing OPCA and Holmes' ataxia to a specific chromosome or chromosomal region should permit the subsequent isolation of the 
gene and the identification of the aberrant protein, with implications for the treatment, the prevention, and early clinical detection of these disorders. In such a study, it is essential to find closely linked genetic markers to the genetic defect and the heterogeneity within the disorder. The pilot linkage study of dominant OPCA was performed by Schut (1951), however, because of a few polymorphic markers used, no markers linked to OPCA gene were found. Since the first report of a possible linkage between dominant OPCA and HLA genes on chromosome 6 (Yakura et al., 1974), a number of workers have studied the linkage of OPCA to HLA and other genetic markers on the short arm of chromosome 6 (6p) (Jackson et al., 1977, 1978; Möller et al., 1978; Wastiaux et al., 1978; Pedersen et al., 1980; Nino et al., 1980; Whittington et al., 1980; Morton et al., 1980; Koeppen et al., 1981; Haines et al., 1984; Kumar et al., 1986). The accumulation of evidence has disclosed that there are HLA-linked and HLA-nonlinked families in OPCA. The OPCA locus in the "HLA-linked families" have now termed SCA1 and assigned to 6p24-p21.3 (Haines and Trofatter, 1986; Wilkie et al., 1987; Kanazawa et al., 1987; Zoghbi et al., 1987, 1988). Recent analyses by Zoghbi et al. $(1987,1988)$ and Wilkie et al. (1987) have demonstrated that recombination frequency between SCA1 and HLA is 0.12 and 0.17 , respectively. The SCA1 is mapped centromeric to the gene for F13A (Wilkie et al., 1987) or telomeric to HLA (Haines and Trofatter, 1986; Kanazawa et al., 1987). Based on the multipoint linkage analysis, Rich et al. (1987) in the same group of Wilkie et al. (1987) have reported that the gene order is HLAA:SCA1:D6S7:F13A:6pter. However, in the present study of 15 HLA-informative OPCA families, we obtained negative evidence for linkage of OPCA to HLA, GLO1, and F13A on $6 \mathrm{p}$, and to any of the genes tested. Although additional OPCA loci cannot be determined, these results indicate probable genetic heterogeneity of OPCA, which is linked and nonlinked to HLA.

In HLA-linked and HLA-nonlinked OPCA families, Pedersen et al. (1980) and Kumar et al. (1986) reported that there are certain differences in the clinical features, such as severity of pyramidal tract involvement, mental decline, or pigmentary retinal degeneration. In his recent classification of dominant OPCA, Harding (1984) discriminated OPCA with pigmentary retinal degeneration from OPCA without it. Correlation of SCA1 to this OPCA classification needs further accumulation of evidences. However, none of the cases in the present study showed pigmentary retinal degeneration. In our OPCA families, only 1 (P.16) out of 15 HLA-informative families were segregated with HLA haplotype. However, pedigree size and the number of subjects of P.16 were too small to ascertain true linkage. Clinically, it was difficult to discriminate this pedigree (P.16) from HLA-nonlinked pedigrees, because clinical manifestations quite similar to P.16 were observed in parts of other HLA-nonlinked pedigrees. In literature, Schut-Swier kindred is the large HLAlinked pedigree which has been genetically studied by Schut (1951), Haines et al. (1984), Haines and Trofatter (1986), Rich et al. (1987), and Wilkie et al. (1987). The clinico-pathological findings of this kindred have also been reported by Schut (1950, 
1951) and Landis et al. (1974). A unique sign of "slow apractic eye movement," as described in this kindred (Landis et al., 1974), is also observed in our P.16 family but also in parts of our HLA-nonlinked families. When compared HLA-nonlinked OPCA pedigrees of ours and those of Wastiaux et al., (1978) to Shut-Swier kindred as well as to the HLA-linked one of Nino et al. (1980), it seems impossible to find any apparent clinical differences between the two types of hereditary OPCA. It is partly because of considerable phenotypic heterogeneity even in single but large pedigrees, as observed in Schut-Swier kindred (Schut, 1950; Landis et al., 1974). At present, it is still uncertain whether there are significant clinical differences, as suggested by several investigators (Pedersen et al., 1980; Kumar et al., 1986), between HLA-linked and -nonlinked OPCA pedigrees. These facts indicate that the phenotypic variation itself cannot be reliable indicator for genetic heterogeneity at OPCA locus.

Autosomal dominant OPCA is currently regarded to be a established clinicopathological entity. However, chiefly because of variation in phenotypes, there have been a matter of debates in the classification of clinical subtypes. Generally speaking, affected individuals in a single family usually tend to have similar clinical features and courses. However, generation anticipation of earlier onset and higher severity of the disease is noted in the descendant generations. Even in a single family, marked variations of clinical features are sometime observed. In addition to this, the extent of pathological involvement also vary from patient to patient in a single family, as well as among separate families. Therefore, a comprehensive picture of the disease could not emerge from the study of insufficient size of affected families, such as most of ours and reported ones. This fact should be taken into consideration when evaluating the correlation of clinical features to previous OPCAHLA linkage results. Furthermore, it indicates that even a single locus of OPCA can produce considerable phenotypic variations. At present, it is not known whether phenotypic heterogeneity within or between affected families is due to multiple loci, multiple alleles, or interaction of modifier genes.

For another prediction for the conflicting results of linkage study of OPCA, pedigree structure, in particular, size and distribution of affected members are considered. However, it is unlikely that nonlinkage to HLA is due to the pedigree structure of affected families, because our families and those of reported ones include sufficient large pedigrees to prove HLA linkage. Even small family pedigrees, theoretically summation of lod scores can prove linkage. The second possibility is that those conflicting results reflect the different recombination rate of investigated families. However, no supportive evidence has been reported. The third possibility in the failure of demonstration of OPCA: HLA linkage is the altered recombination rate in geographically isolated and genetically homogeneous populations, particularly such as Japanese. Recently demonstrated linkage of F13A:HLA (Wong et al., 1988) has not been reconfirmed not only in our study but also in the extensive study of Nishigaki et al. (1986), even by using sufficient number of pedigrees. The discrep- 
ancy of this linkage data between these of Japanese and those in English literature implies that, for instance, OPCA:HLA nonlinkage obtained in our study reflects the potentially higher recombination rate occurring in Japanese than expected. Although we took this possibility into account, far negative values observed in the paternal lod scores for HLA:OPCA linkage than those for F13A:HLA linkage seem against this possibility (Table 4). When taken our results and previous genetic linkage studies into consideration, it is most likely at the present time that there is genetic heterogeneity in dominant OPCA, as suggested by several authors both genetically and clinically (Wastiaux et al., 1978; Pedersen et al., 1980; Whittington et al., 1980; Morton et al., 1980; Koeppen et al., 1981; Kumar et al., 1986). If there is genetic heterogeneity in OPCA, it implies that simple summing of lod scores in OPCA linkage studies of different families will make the linkage obscure. For this reason, it is reliable, if possible, to obtain linkage information from a single large family rather than from many different small families, as already mentioned (Ott, 1985). Until now, no markers other than HLA have been documented to be linked to SCA1 locus by two-point linkage analysis. In order to solve the discrepancy in OPCA:HLA linkage studies and also to confirm the possible genetic heterogeneity of OPCA, it is necessary to find a set of polymorphic $6 \mathrm{p}$ markers other than HLA which must be more tightly linked to SCA1 locus than HLA. In addition, ruling out of the localization of HLA-nonlinked OPCA locus on chromosome $6 \mathrm{p}$ by systematic linkage study is essential to prove genetic heterogeneity of OPCA.

In contrast to OPCA, Holmes' ataxia is a distinct clinico-pathological entity in hereditary SCA. However, systematic genetic linkage studies of Holmes' ataxia have not been performed. Koeppen et al. (1981) investigated GLO1, Bf, and HLA in an affected family (pedigree 1 in their report), but found no evidence of linkage. Our results have not indicated any conclusive evidence of linkage between Holmes' ataxia and the test markers. In order to estimate the location of the locus of Holmes' ataxia, it is necessary to collect further informative families as well as to perform continuous genetic linkage studies.

Acknowledgment We express our gratitude first to the members of SCA families who understood our purpose and cooperated to this investigation. We are grateful to Dr. J. Ott (Columbia University) for sending us LIPED program. We thank to Drs., Kazuto Yoshida, Yasuharu Aimoto (Kusiro Rosai Hospital), Fumio Moriwaka, Naoto Fujiki (Department of Neurology, School of Medicine, Hokkaido University), Mutsuo Naganuma, Sizuki Doi (Sapporo Minami National Sanatory Hospital), Osamu Yahara (Department of Internal Medicine, Asahikawa Medical College), Tetsuo Yanagihara, Kohji Hamada, Toshiyuki Fukazawa (Hokuyukai Neurological Hospital), and others who kindly gave a chance to examine patients. We also express our thanks to Drs. Hitoshi Satoh, Shuichi Abe (Chromosomal Research Unit, Faculty of Science, Hokkaido University), and Sigenori Ikemoto (Department of Forensic Medicine. Jichi Medical College) for their kind technical as well as academical advices and assistance. Drs. Sadami Sekiguchi and Hisami Ikeda (Hokkaido Red Cross Blood Center) have kindly cooperated in typing the blood groups. Dr. J. Aparicio is also gratefully acknowledged for reviewing our English manuscript. 


\section{REFERENCES}

Board, P.G. 1979. Genetic polymorphism of the A subunit of human coagulating factor XIII. Am. J. Hum. Genet. 31: 116-124.

Fujii, M., Fukatsu, R., Takahata, N., and Kawata, H. 1987. A unique family of hereditary ataxia: clinico-pathological study of an autopsied case (Japanese abstract). Clin. Neurol. (Tokyo) 27: 781 .

Haines, J.L., Schut, L.J., Weikamp, L.R., Thayer, M., and Anderson, E. 1984. Spinocerebellar ataxia in a large kindred: age at onset, reproduction, and genetic linkage studies. Neurology (Cleveland) 34: 1542-1548.

Haines, J.L. and Trofatter, J.A. 1986. Multipoint linkage analysis of spinocerebeliar ataxia and markers on chromosome 6. Genet. Epidemiol. 3: 399-405.

Harding, A.E. 1984. Autosomal dominant cerebellar ataxia. In The Hereditary Ataxias and Related Disorders, Churchill Livingstone, New York, pp. 129-165.

Harris, H. and Hopkinson, D.A. 1976. Handbook of Enzyme Electrophoresis in Human Genetics, North-Holland, Oxford.

Jackson, J.F., Currier, R.D., Terasaki, P.I., and Morton, N.E. 1977. Spinocerebellar ataxia and HLA linkage. N. Engl. J. Med. 296: 1138-1141.

Jackson, J.F., Whittington, J.E., Currier, R.D., Terasaki, P., Morton, N.E., and Keats, B.J. 1978. Genetic linkage and spinocerebellar ataxia. Adv. Neurol. 21 : 315-318.

Kanazawa, I., Yoshizawa, K., Kondo, I., and Ikemoto, S. 1987. The dominantly inherited spinocerebellar ataxia (SCA1) locus maps distally from HLA on chromosome 6. Cytogenet. Cell Genet. 46: 636 (HGM9 abstract).

Koeppen, A.H., Goedde, H.W., Hiller, C., Hirth, L., and Benkmann, H.-G. 1981. Hereditary ataxia and the sixth chromosome. Arch. Neurol. 38: 158-164.

Kudoh, J., Ikeda, T., and Takahata, N. 1986. A clinico-pathological study of slow saccades and sleep disturbances-Two attopsy cases with hereditary spinocerebellar degeneration. Clin. Neurol. (Tokyo) 26: 601-611.

Kumar, D., Blank, C.E., and Gelsthorpe, K. 1986. Hereditary cerebellar ataxia and genetic linkage with HLA. Hum. Genet. 72: 327-332.

Landis, D.M.D., Rosenberg, R.N., Landis, S.C., Schut, L., and Nyhan, W.L. 1974. Olivopontocerebellar degeneration. Clinical and ultrastructural abnormalities. Arch. Neurol. 31: 295307.

Möller, E., Hindfelt, B., and Olsson, J.-E. 1978. HLA-determination in families with hereditary ataxia. Tissue Antigens 12: 357-366.

Morton, N.E., Lalouel, J.-M., Jackson, J.F., Currier, R.D., and Yee, S. 1980. Linkage studies in spinocerebellar ataxia (SCA). Am. J. Med. Genet. 6: 251--257.

Nino, H.E., Noreen, H.J., Dubey, D.P., Resch, J.A., Namboodiri, K., Elston, R.C., and Yunis, E. 1980. A family with hereditary ataxia: HLA typing. Neurology (N.Y.) 3: 12-20.

Nishigaki, T., Tokunaga, K., Ishii, C., Omoto, K., Akaza, T., Akiyama, N., Juji, T., Murata, S., Naito, S., Saji, H., Satoh, H., Shiraki, T., Tokunaga, K., and Yasuda, N. 1986. No evidence for linkage between the loci for coagulating factor XIII-A and HLA. Hum. Genet. 72: 266-267.

Olaisen, B., Sakaguchi, A., and Naylor, S.L. 1987. Report of the committee on the genetic constitution of chromosome 5 and 6. Cytogenet. Cell Genet. 46: 147-169.

Ott, J. 1974. Estimation of the recombination fraction in human pedigrees: Efficient computation of the likelihood for human linkage studies. Am. J. Hum. Genet. 26: 588-597.

Ott, J. 1978. A simple scheme for the analysis of HLA linkages in pedigrees. Ann. Hum. Genet. 42: $255-257$.

Ott, J. 1985. Few large or many small families? In Analysis of Human Genetic Linkage, The Johns Hopkins University Press, Baltimore, p. 55. 
Pedersen, L., Platz, P., Ryder, L.P., Lamm, L.U., and Dissing, J. 1980. A linkage study of hereditary ataxias and related disorders. Evidence of heterogeneity of dominant cerebellar ataxia. Hum. Genet. 54: 371-383.

Rich, S.S., Wilkie, P., Shut, L., Vance, G., and Orr, H.T. 1987. Spinocerebellar ataxia: Localization of an autosomal dominant locus between two markers on human chromosome 6. Am. J. Hum. Genet. 41: 524-531.

Rosenberg, R.N. 1986. Spinocerebellar degenerations. In Neurogenetics. Principles and Practice, Raven Press, New York, pp. 109-134.

Schut, C.J.W. 1950. Hereditary ataxia. Clinical study through six generations. Arch. Neurol. Psychiat. 63: 535-568.

Schut, C.J.W. 1951. Hereditary ataxia. A survey of certain clinical, pathological and genetic features with linkage data on five additional hereditary factors. Am. J. Hum. Genet. 3: 93-110.

Wastiaux, J.P., Lamoureux, G., Bouchard, J.P., Durivage, A., Barbeau, C., and Barbeau, A. 1978. HLA and complement typing in olivo-ponto-cerebellar atrophy, Can. J. Neurol. Sci. 5: 75-81.

Whittington, J.E., Keats, B.J.B., Jackson, J.F., Currier, R.D., and Terasaki, P.I. 1980. Linkage studies on glyoxalase I (GLO), pepsinogen (PG), spinocerebellar ataxia (SCA1), and HLA. Cytogenet. Cell Genet. 28: 145-150.

Wilkie, P., Rich, S., Schut, L., and Orr, H. 1987. Spinocerebellar ataxia: localization of the autosomal dominant HLA-linked form between two markers on human chromosome 6. Cytogenet. Cell Genet. 46: 715 (HGM9 abstract).

Wong, P., Komarnicki, L., Schroeder, M.L., Lewis, M., Kaita, H., Philipps, S., Stranc, L., and McAlpine, P.J. 1988. Analysis for linkage between F13A and three chromosome 6 marker loci: evidence for 6pter:F13A:HLA:GLO1:cen gene order. Hum. Genet. 79: 228-230.

Yakura, H., Wakisaka, A., Fujimoto, S., and Itakura, K. 1974. Hereditary ataxia and HL-A genotypes. N. Engl. J. Med. 291: 154-155.

Zoghbi, H.Y., Pollack, M.S., Ferrell, R.E., Daiger, S.P., Lyons, L.A., O’Brien, W.E., and Beaudet, A.L. 1987. Linkage studies in spinocerebellar ataxia (SCA1). Cytogenet. Cell Genet. 46: 727 (HGM9 abstract).

Zoghbi, H.Y., Pollack, M.S., Lyons, L.A., Ferrell, R.E., Daiger, S.P., and Beaudet, A.L. 1988. Spinocerebellar ataxia: variable age of onset and linkage to human leukocyte antigen in a large kindred. Ann. Neurol. 23: 580-584. 